\title{
ONE-RELATOR GROUPS AND ALGEBRAS RELATED TO POLYHEDRAL PRODUCTS
}

\author{
JELENA GRBIĆ, MARINA ILYASOVA, TARAS PANOV, AND GEORGE SIMMONS
}

\begin{abstract}
We link distinct concepts of geometric group theory and homotopy theory through underlying combinatorics. For a flag simplicial complex $K$, we specify a necessary and sufficient combinatorial condition for the commutator subgroup $R C_{K}^{\prime}$ of a right-angled Coxeter group, viewed as the fundamental group of the real moment-angle complex $\mathcal{R}_{K}$, to be a one-relator group; and for the Pontryagin algebra $H_{*}\left(\Omega \mathcal{Z}_{K}\right)$ of the moment-angle complex to be a one-relator algebra. We also give a homological characterisation of these properties. For $R C_{K}^{\prime}$, it is given by a condition on the homology group $H_{2}\left(\mathcal{R}_{K}\right)$, whereas for $H_{*}\left(\Omega \mathcal{Z}_{K}\right)$ it is stated in terms of the bigrading of the homology groups of $\mathcal{Z}_{K}$.
\end{abstract}

\section{INTRODUCTION}

Let $K$ be a flag simplicial complex on vertex set $[m]=\{1, \ldots, m\}$ and $K^{1}$ be its 1-skeleton. The right-angled Coxeter group corresponding to $K$ is defined as the group $R C_{K}$ with generators $g_{1}, \ldots, g_{m}$ for each vertex in $K$ and relations $g_{i}^{2}=1$ and $g_{i} g_{j}=g_{j} g_{i}$ whenever $\{i, j\} \in K^{1}$. Right-angled Coxeter groups are interesting from a geometric point of view because they arise from reflections in the facets of right-angled polyhedra in hyperbolic space.

For a given group $G$, we denote by $G^{\prime}$ the commutator subgroup of $G$. The real moment-angle complex $\mathcal{R}_{K}=\left(D^{1}, S^{0}\right)^{K}$ associated with a flag complex $K$ is a finite-dimensional aspherical space whose fundamental group is the commutator subgroup $R C_{K}^{\prime}$ of the right-angled Coxeter group $R C_{K}$. In [15] it was shown that $R C_{K}^{\prime}=\pi_{1}\left(\mathcal{R}_{K}\right)$ is free if and only if $K^{1}$ is a chordal graph. A graph is called chordal if each of its cycles with 4 or more vertices has a chord, an edge joining two vertices that are not adjacent in the cycle. Furthermore, for arbitrary flag $K$, a minimal generating set for $R C_{K}^{\prime}$ was given in terms of iterated commutators of the generators of $R C_{K}$ [15, Theorem 4.5].

Another space associated with a simplicial complex $K$ is the moment-angle complex $\mathcal{Z}_{K}=\left(D^{2}, S^{1}\right)^{K}$. Throughout this paper, all homology groups are considered with coefficients in $\mathbb{Z}$, unless otherwise stated. The Pontryagin algebra $H_{*}\left(\Omega \mathcal{Z}_{K}\right)$, was studied in [7] when $K$ is a flag complex. It was shown that $H_{*}\left(\Omega \mathcal{Z}_{K}\right)$ is a graded free associative algebra if and only if the 1-skeleton $K^{1}$ is a chordal graph. Furthermore, a minimal generating set for $H_{*}\left(\Omega \mathcal{Z}_{K}\right)$ with flag $K$ was given in [7, Theorem 4.3] in terms of iterated commutators.

Therefore, for both $\mathcal{R}_{K}$ and $\mathcal{Z}_{K}$ the algebraic freeness property, that is, that $\pi_{1}\left(\mathcal{R}_{K}\right)$ and $H_{*}\left(\Omega \mathcal{Z}_{K}\right)$ are free as groups and algebras, respectively, is characterised by the same combinatorial condition. More precisely, these algebraic objects are free if and only if the 1-skeleton $K^{1}$ of the simplicial complex $K$ is a chordal graph. The question of $H_{*}\left(\Omega \mathcal{Z}_{K}\right)$ being a free associative algebra is related to the Golodness

The third author was supported by the Russian Foundation for Basic Research grant no. 2001-00675. The authors thank the Fields Institute for Research in Mathematical Sciences for the opportunity to work on this research project during the Thematic Program on Toric Topology and Polyhedral Products. 
property of a simplicial complex $K$. A simplicial complex $K$ is Golod if all cup products and higher Massey products vanish in $H^{*}\left(\mathcal{Z}_{K}\right)$. In [7, Theorem 4.6] it was proved that a flag simplicial complex $K$ is Golod if and only if $K^{1}$ is a chordal graph.

In this paper we study other properties of objects naturally arising in geometric group theory and homotopy theory that have the same combinatorial characterisation. In particular, we describe a combinatorial condition on a flag complex $K$ under which $\pi_{1}\left(\mathcal{R}_{K}\right)$ is a one-relator group, and $H_{*}\left(\Omega \mathcal{Z}_{K}\right)$ is a one-relator algebra. A 1-dimensional simplicial complex $C_{p}$ that is the boundary of a $p$-gon is called a $p$-cycle. In [7] it was shown that when $K$ is a 5 -cycle then there is only one relation between the 10 multiplicative generators of $H_{*}\left(\Omega \mathcal{Z}_{K}\right)$; while in [16], a single relation was again found between the 34 multiplicative generators of $H_{*}\left(\Omega \mathcal{Z}_{K}\right)$ when $K$ is a 6-cycle. Similarly, in [15], it was noted that if $K$ is a $p$-cycle for $p \geqslant 4$, then $\pi_{1}\left(\mathcal{R}_{K}\right)$ is a one-relator group. The one-relator condition places strong restrictions on the form of $K$, and our main combinatorial characterisation is the following.

Theorem 1.1. Let $K$ be a flag simplicial complex. Then $\pi_{1}\left(\mathcal{R}_{K}\right)$ and $H_{*}\left(\Omega \mathcal{Z}_{K}\right)$ have exactly one relation if and only if the following combinatorial condition holds

$$
K=C_{p} \text { or } K=C_{p} * \Delta^{q} \text { for } p \geqslant 4, q \geqslant 0
$$

where $C_{p}$ is a p-cycle, $\Delta^{q}$ is a q-simplex and $*$ denotes the join of simplicial complexes.

For $\mathcal{R}_{K}$ this is proved in Theorem 3.2 and for $\mathcal{Z}_{K}$ in Theorem 5.1. The proofs of Theorem 3.2 and Theorem 5.1 are completely different in character. For Theorem 3.2, the key argument comes from geometric group theory. When $K=C_{p}$ or $K=C_{p} * \Delta^{q}$ for $q \geqslant 0$, the space $\mathcal{R}_{K}$ is homeomorphic to the product $S_{g} \times D^{q+1}$, where $S_{g}$ is a closed orientable surface of genus $g=(p-4) 2^{p-3}+1$ and $D^{q+1}$ is a $(q+1)$-dimensional disc, and therefore its fundamental group is a one-relator surface group. The converse statement is proved using the Lyndon Identity Theorem [12] (see [6, Theorem 2.1]) because the group $\pi_{1}\left(\mathcal{R}_{K}\right)=R C_{K}^{\prime}$ is torsion-free.

To prove Theorem 5.1, we study the simply connected space $\Omega \mathcal{Z}_{K}$ using homotopytheoretical methods. When $K=C_{p}$ or $K=C_{p} * \Delta^{q}$ for $q \geqslant 0$, by a result of McGavran [13], there is a homotopy equivalence

$$
\mathcal{Z}_{K} \simeq \#_{k=3}^{p-1}\left(S^{k} \times S^{p+2-k}\right) \#(k-2)\left(\begin{array}{l}
p-2 \\
k-1
\end{array}\right)
$$

where \# denotes the connected sum operation on manifolds. Beben and $\mathrm{Wu}[4]$ computed the algebra $H_{*}\left(\Omega X ; \mathbb{Z}_{p}\right), p$ prime, where $X$ is a highly-connected manifold obtained by attaching a single cell to a space $Y$ which has the homotopy type of a double suspension. This implies that $H^{*}\left(Y ; \mathbb{Z}_{p}\right)$ has no non-trivial cup products, which places sufficient restrictions on $H^{*}\left(X ; \mathbb{Z}_{p}\right)$ so that $H_{*}\left(\Omega X ; \mathbb{Z}_{p}\right)$ can be studied via a homology Serre spectral sequence. We adapt the Beben-Wu method to study the integral Pontryagin algebra of an arbitrary connected sum of sphere products

$$
M=\#_{i=1}^{k}\left(S^{d_{i}} \times S^{d-d_{i}}\right)
$$

where $d_{i} \geqslant 2$ and $d \geqslant 4$. In this case, the Beben-Wu method reduces to the Adams-Hilton model and the highly-connectedness assumption can be dropped. In Proposition 4.1, we prove that the integral Pontryagin algebra $H_{*}(\Omega M)$ is isomorphic as a Hopf algebra to the quotient of a graded free associative algebra by a single relation. Proposition 4.1 implies that when $K=C_{p}$ or $K=C_{p} * \Delta^{q}$ for $q \geqslant 0$, $H_{*}\left(\Omega \mathcal{Z}_{K}\right)$ is a one-relator algebra. We compute the Poincaré series $P\left(H_{*}\left(\Omega \mathcal{Z}_{K}\right) ; t\right)$ explicitly in Proposition 4.2.

We extend the equivalences of Theorem 1.1 by determining an equivalent homological criteria on $\mathcal{R}_{K}$ and $\mathcal{Z}_{K}$. For $\mathcal{R}_{K}$, the combinatorial condition (*) is 
equivalent to the homological condition $H_{2}\left(\mathcal{R}_{K}\right)=\mathbb{Z}$, and this is proved in Theorem 3.2. The homology groups of $\mathcal{Z}_{K}$ have a natural bigrading, see [5, § 4.4]. The combinatorial condition $(*)$ is then equivalent to the homological condition

$$
H_{2-j, 2 j}\left(\mathcal{Z}_{K}\right)= \begin{cases}\mathbb{Z} & \text { if } j=p \\ 0 & \text { otherwise }\end{cases}
$$

This is proved in Theorem 5.1.

Although the homotopy type of a moment-angle complex $\mathcal{Z}_{K}$ is not accessible in general, various homotopy-theoretical concepts can be described if $K$ is a flag complex. Moreover, many of these homotopy-theoretical characterisations of $\mathcal{Z}_{K}$ are equivalent. For example, for $K$ a flag complex, $\mathcal{Z}_{K}$ having the homotopy type of a wedge of spheres is equivalent to $\mathcal{Z}_{K}$ being a co-H space and these concepts are equivalent to $K$ being Golod. In this paper, we show that $H_{*}\left(\Omega \mathcal{Z}_{K}\right)$ is a onerelator algebra if and only if $\mathcal{Z}_{K}$ has the homotopy type of a connected sum of sphere products, with two spheres in each product. Additionally, these properties are closely related to $K$ being minimally non-Golod, see [11], and we summarise this relationship in Proposition 5.6.

We note that despite the similarity of the results for the moment-angle complex $\mathcal{Z}_{K}$ and its real analogue $\mathcal{R}_{K}$ when $K$ is flag, the techniques used in proofs differ significantly. For the case of $\mathcal{Z}_{K}$, homotopy-theoretical methods are more prevalent, whereas the case of $\mathcal{R}_{K}$ requires the use of methods in combinatorial and geometric group theory. Given a homotopy-theoretical result related to $\mathcal{Z}_{K}$, one could predict the corresponding group-theoretical result for $\mathcal{R}_{K}$, but it is an open and challenging problem to find a systematic way of translating these results directly. This is a problem of interest to both topologists and group theorists.

For non-flag $K$, all homotopy-theoretical characterisations of moment-angle complexes $\mathcal{Z}_{K}$ are more complex. For example, for an arbitrary Golod complex $K$, the moment-angle complex $\mathcal{Z}_{K}$ is not necessarily a co-H space [10] and its cohomology can contain torsion [7]. Furthermore, describing the Pontryagin algebra $H_{*}\left(\Omega \mathcal{Z}_{K}\right)$, and in particular determining the class of $K$ for which it is a free or one-relator algebra, is considerably harder in the non-flag case. The problem of determining those $K$ which are Golod or minimally non-Golod is also more involving, and in general distinct from studying $H_{*}\left(\Omega \mathcal{Z}_{K}\right)$. At the end of Section 5 we expand on the distinction between the properties of $K$ being minimally non-Golod and $H_{*}\left(\Omega \mathcal{Z}_{K}\right)$ being a one-relator algebra. This complexity is also seen in the real case. In the non-flag case, the real moment-angle complex $\mathcal{R}_{K}$ is not aspherical, so its topology is not determined by its fundamental group. Therefore, the question of describing $H_{*}\left(\Omega \mathcal{R}_{K}\right)$ does not lie entirely within combinatorial group theory.

\section{Preliminaries}

Let $K$ be a simplicial complex on the set $[m]=\{1,2, \ldots, m\}$, that is, $K$ is a collection of subsets $I \subseteq[\mathrm{m}]$ such that for any $I \in K$ all subsets of $I$ also belong to $K$. We always assume that $K$ contains $\emptyset$ and all singletons $\{i\} \in[m]$.

Let

$$
(\mathbf{X}, \mathbf{A})=\left\{\left(X_{1}, A_{1}\right), \ldots,\left(X_{m}, A_{m}\right)\right\}
$$

be a sequence of $m$ pairs of pointed topological spaces, $p t \in A_{i} \subseteq X_{i}$. For each subset $I \subseteq[m]$, we set

$$
(\mathbf{X}, \mathbf{A})^{I}=\left\{\left(x_{1}, \ldots, x_{m}\right) \in \prod_{k=1}^{m} X_{k} \mid x_{k} \in A_{k} \text { for } k \notin I\right\}
$$


and define the polyhedral product of $(\mathbf{X}, \mathbf{A})$ over the complex $K$ as

$$
(\mathbf{X}, \mathbf{A})^{K}=\bigcup_{I \in K}(\mathbf{X}, \mathbf{A})^{I}=\bigcup_{I \in K}\left(\prod_{i \in I} X_{i} \times \prod_{i \notin I} A_{i}\right) \subseteq \prod_{k=1}^{m} X_{k}
$$

In the case when $X_{i}=X$ and $A_{i}=A$ for all $i$ we use the notation $(X, A)^{K}$ for $(\mathbf{X}, \mathbf{A})^{K}$.

\section{Example 2.1.}

1. Let $(X, A)=\left(D^{1}, S^{0}\right)$, where $D^{1}$ is the closed interval $[-1,1]$ and $S^{0}$ is its boundary $\{-1,1\}$. The polyhedral product $\left(D^{1}, S^{0}\right)^{K}$ is known as the real momentangle complex and is denoted by $\mathcal{R}_{K}$,

$$
\mathcal{R}_{K}=\left(D^{1}, S^{0}\right)^{K}=\bigcup_{I \in K}\left(D^{1}, S^{0}\right)^{I} .
$$

Note that $\mathcal{R}_{K}$ is a cubic subcomplex in the cube $\left(D^{1}\right)^{m}=[-1,1]^{m}$.

2. Let $(X, A)=\left(D^{2}, S^{1}\right)$, where $D^{2}$ is the closed unit disc and $S^{1}$ is its boundary. The polyhedral product $\left(D^{2}, S^{1}\right)^{K}$ is known as the moment-angle complex and is denoted by $\mathcal{Z}_{K}$. If $D^{2}$ is considered as a $C W$-complex with one cell in each dimension zero, one and two, then the moment-angle complex $\mathcal{Z}_{K}$ is a $C W$-subcomplex of the $C W$-product complex $\left(D^{2}\right)^{m}$.

3. Let $(X, A)=\left(\mathbb{C} P^{\infty}, p t\right)$. The polyhedral product $\left(\mathbb{C} P^{\infty}, p t\right)^{K}$ is known as the Davis-Januszkiewicz space and is denoted by $D J_{K}$.

For any subset $J \subseteq[m]$, the corresponding full subcomplex of $K$ is defined by

$$
K_{J}=\{I \in K \mid I \subseteq J\} .
$$

The homology groups of the moment-angle complex $\mathcal{Z}_{K}$ have a natural bigrading arising from the bigrading in the $\mathrm{CW}$-structure of $\mathcal{Z}_{K}$, see $[5, \S 4.4]$,

$$
H_{k}\left(\mathcal{Z}_{K}\right) \cong \bigoplus_{-i+2 j=k} H_{-i, 2 j}\left(\mathcal{Z}_{K}\right)
$$

The bigraded components $H_{-i, 2 j}\left(\mathcal{Z}_{K}\right)$ can be described through the reduced simplicial homology groups of full subcomplexes $K_{J}$ using Hochster's theorem, see [5, Theorem 4.5.8],

$$
H_{-i, 2 j}\left(\mathcal{Z}_{K}\right) \cong \bigoplus_{J \subseteq[m],|J|=j} \widetilde{H}_{j-i-1}\left(K_{J}\right)
$$

Similarly, the homology groups of the real moment-angle complex $\mathcal{R}_{K}$ are given by

$$
H_{k}\left(\mathcal{R}_{K}\right) \cong \bigoplus_{J \subseteq[m]} \widetilde{H}_{k-1}\left(K_{J}\right)
$$

for any $k \geqslant 0$, see $[5, \S 4.5]$.

A missing face of $K$ is a subset $I \subseteq[m]$ such that $I$ is not a simplex of $K$, but every proper subset of $I$ is a simplex of $K$. A simplicial complex $K$ is called a flag complex if each of its missing faces consists of two vertices, that is, any set of vertices of $K$ which are pairwise connected by edges spans a simplex. A clique of a graph $\Gamma$ is a subset $I$ of vertices pairwise connected by edges. For a graph $\Gamma$, we define the clique complex of $\Gamma$ as the simplicial complex obtained by filling in each clique of $\Gamma$ by a simplex. Each flag complex $K$ is the clique complex of its 1-skeleton $\Gamma=K^{1}$.

A graph $\Gamma$ is called chordal if each of its cycles with 4 or more vertices has a chord, an edge joining two vertices that are not adjacent in the cycle. A p-cycle is 
the same as the boundary of a $p$-gon. It is a chordal graph only when $p=3$. The simplicial complex which is a $p$-cycle is denoted by $C_{p}$.

If $K=C_{p}, p \geqslant 4$, by a result of McGavran [13], there is a homeomorphism

$$
\mathcal{Z}_{K} \cong \#_{k=3}^{p-1}\left(S^{k} \times S^{p+2-k}\right)^{\#(k-2)\left(\begin{array}{l}
p-2 \\
k-1
\end{array}\right)} .
$$

The corresponding real moment-angle complex $\mathcal{R}_{K}$ is an orientable surface of genus $1+(p-4) 2^{p-3}$, see [5, Proposition 4.1.8].

The algebra $H_{*}\left(\Omega \mathcal{Z}_{K}\right)$ was studied in $[14,7]$. The homotopy fibration $\mathcal{Z}_{K} \rightarrow$ $D J_{K} \rightarrow\left(\mathbb{C} P^{\infty}\right)^{m}$ gives rise to a short exact sequence of Hopf algebras

$$
1 \longrightarrow H_{*}\left(\Omega \mathcal{Z}_{K}\right) \longrightarrow H_{*}\left(\Omega D J_{K}\right) \stackrel{\mathrm{Ab}}{\longrightarrow} \Lambda\left[u_{1}, \ldots, u_{m}\right] \longrightarrow 0
$$

where $\mathrm{Ab}$ is the "abelianisation" homomorphism to the graded commutative algebra $\Lambda\left[u_{1}, \ldots, u_{m}\right]=H_{*}\left(\Omega\left(\mathbb{C} P^{\infty}\right)^{m}\right)$ with $\operatorname{deg} u_{i}=1$. The algebra $H_{*}\left(\Omega \mathcal{Z}_{K}\right)$ can be viewed as the commutator subalgebra of $H_{*}\left(\Omega D J_{K}\right)$. Let $[a, b]=a b+$ $(-1)^{\operatorname{deg} a \operatorname{deg} b+1} b a$ denote the graded Lie commutator of the elements $a$ and $b$. In the case that $K$ is flag, there is an algebra isomorphism [14, Theorem 9.3]

$$
H_{*}\left(\Omega D J_{K}\right) \cong T\left(u_{1}, \ldots, u_{m}\right) /\left\langle u_{i}^{2},\left[u_{i}, u_{j}\right] \text { if }\{i, j\} \in K\right\rangle
$$

where $T\left(u_{1}, \ldots, u_{m}\right)$ is a graded free associative algebra and $\operatorname{deg} u_{i}=1$. A minimal multiplicative generating set for $H_{*}\left(\Omega \mathcal{Z}_{K}\right)$ is given as in [7, Theorem 4.3]. Namely, $H_{*}\left(\Omega \mathcal{Z}_{K}\right)$ is multiplicatively generated by $\sum_{J \subseteq[m]} \operatorname{rank} \widetilde{H}_{0}\left(K_{J}\right)$ iterated commutators of the form

$$
\left[u_{j}, u_{i}\right],\left[u_{k_{1}},\left[u_{j}, u_{i}\right]\right], \ldots,\left[u_{k_{1}},\left[u_{k_{2}}, \ldots,\left[u_{k_{l-2}},\left[u_{j}, u_{i}\right]\right] \ldots\right]\right]
$$

where $k_{1}<k_{2}<\cdots<k_{l-2}<j>i, k_{s} \neq i$ for any $s$ and $i$ is the smallest vertex in a connected component of $K_{\left\{k_{1}, \ldots, k_{l-2}, j, i\right\}}$ not containing $j$. Additionally, it was shown in [7, Theorem 4.6] that $H_{*}\left(\Omega \mathcal{Z}_{K}\right)$ is a free associative algebra if and only if the graph $K^{1}$ is chordal, in which case $\mathcal{Z}_{K}$ is homotopy equivalent to a wedge of spheres.

Parallel results for the real moment-angle complex $\mathcal{R}_{K}$ were obtained in [15] in the group-theoretical setting. Let $(g, h)=g^{-1} h^{-1} g h$ denote the group commutator of elements $g$ and $h$.

The right-angled Coxeter group $R C_{K}$ corresponding to $K$ is defined by

$$
R C_{K}=F\left(g_{1}, \ldots, g_{m}\right) /\left(g_{i}^{2},\left(g_{i}, g_{j}\right) \text { if }\{i, j\} \in K\right)
$$

where $F\left(g_{1}, \ldots, g_{m}\right)$ is a free group with $m$ generators. Note that $R C_{K}$ depends only on the 1-skeleton $\Gamma=K^{1}$, which is a graph.

Recall that a path-connected space $X$ is aspherical if $\pi_{i}(X)=0$ for $i \geqslant 2$. An aspherical space $X$ is an Eilenberg-Mac Lane space $K(\pi, 1)$ with $\pi=\pi_{1}(X)$. The following facts relating the real moment-angle complex $\mathcal{R}_{K}$ to the right-angle Coxeter group $R C_{K}$ are known, see, for example [15, Corollary 3.4]):

(i) $\pi_{1}\left(\mathcal{R}_{K}\right)$ is isomorphic to the commutator subgroup $R C_{K}^{\prime}$;

(ii) $\mathcal{R}_{K}$ is aspherical if and only if $K$ is flag.

Therefore, in the flag case the algebra $H_{*}\left(\Omega \mathcal{R}_{K}\right)$ reduces to the non-abelian group $H_{0}\left(\Omega \mathcal{R}_{K}\right)=R C_{K}^{\prime}$ and the analogue of $(6)$ is the short exact sequence of groups

$$
1 \longrightarrow R C_{K}^{\prime} \longrightarrow R C_{K} \stackrel{\mathrm{Ab}}{\longrightarrow}\left(\mathbb{Z}_{2}\right)^{m} \longrightarrow 0
$$

where $\mathbb{Z}_{2}$ is an elementary abelian 2-group and $\mathrm{Ab}$ is the abelianisation homomorphism.

By analogy with [7], the following combinatorial criterion was obtained in [15, Corollary 4.4]: the commutator subgroup $R C_{K}^{\prime}$ is a free group if and only if the graph $K^{1}$ is chordal. 
An explicit minimal generator set for the commutator subgroup $R C_{K}^{\prime}$ is described in $\left[15\right.$, Theorem 4.5]. It consists of $\sum_{J \subseteq[m]} \operatorname{rank} \widetilde{H}_{0}\left(K_{J}\right)$ nested commutators

$$
\left(g_{j}, g_{i}\right),\left(g_{k_{1}},\left(g_{j}, g_{i}\right)\right), \ldots,\left(g_{k_{1}},\left(g_{k_{2}}, \ldots,\left(g_{k_{l-2}},\left(g_{j}, g_{i}\right)\right) \ldots\right)\right)
$$

where $k_{1}<k_{2}<\cdots<k_{l-2}<j>i, k_{s} \neq i$ for any $s$, and $i$ is the smallest vertex in a connected component of $K_{\left\{k_{1}, \ldots, k_{l-2}, j, i\right\}}$ not containing $j$.

\section{One-Relator Groups}

A group $G$ is called a one-relator group if $G$ is not a free group and can be presented with a generating set with a single relation.

Let $G$ be a one-relator group, that is, $G=F / R$, where $F=F\left(x_{1}, \ldots, x_{l}\right)$ is a free group and $R$ is the smallest normal subgroup in $F$ generated by relation $r$. Consider the space

$$
Y(G)=\left(\bigvee_{i=1}^{l} S_{i}^{1}\right) \cup_{\bar{r}} e^{2}
$$

obtained by attaching a 2-cell to a wedge of circles via a map $\bar{r}: S^{1} \rightarrow \bigvee S_{i}^{1}$ corresponding to the element $r \in F$.

Recall that all homology groups are considered with coefficients in $\mathbb{Z}$. The homology groups of $Y(G)$ are described as follows.

Proposition 3.1. $H_{k}(Y(G))=0$ for $k \geqslant 3, H_{1}(Y(G))=\mathbb{Z}^{l}$ and

$$
H_{2}(Y(G))= \begin{cases}\mathbb{Z} & \text { if } r \in[F, F] \\ 0 & \text { otherwise. }\end{cases}
$$

Lyndon [12] studied cohomology theory of groups with a single relation by considering the corresponding space $Y(G)$. Dyer and Vasquez [6] gave an equivalent formulation of the Lyndon Identity Theorem in the following form (see [6, Theorem 2.1]l): if $G$ is a one-relator group with relation $r$ which is not a proper power, that is, $r \neq u^{n}$ for $n>1$, then $Y(G)$ is a $K(G, 1)$-space.

Under the conditions of the Lyndon Identity Theorem, we have $H_{k}(G ; \mathbb{Z})=$ $H_{k}(Y(G) ; \mathbb{Z})$, that is, the homological dimension of $G$ is at most 2 .

Theorem 3.2. Let $K$ be a flag simplicial complex on $[m]$. The following conditions are equivalent:

(a) $\pi_{1}\left(\mathcal{R}_{K}\right)=R C_{K}^{\prime}$ is a one-relator group;

(b) $H_{2}\left(\mathcal{R}_{K}\right)=\mathbb{Z}$;

(c) $K=C_{p}$ or $K=C_{p} * \Delta^{q}$ for $p \geqslant 4$ and $q \geqslant 0$, where $C_{p}$ is a $p$-cycle, $\Delta^{q}$ is a q-simplex, and $*$ denotes the join of simplicial complexes.

If any one of these conditions is met, we have $H_{k}\left(\mathcal{R}_{K}\right)=0$ for $k \geqslant 3$.

Proof. (c) $\Rightarrow(\mathrm{b})$. This implication follows from the implications below, but we include an independent proof as it is simple and illustrative. Suppose that $K=C_{p}$ or $K=C_{p} * \Delta^{q}$ for $p \geqslant 4$ and $q \geqslant 0$. Let the $p$-cycle $C_{p}$ be supported on the set of vertices $I=\left\{i_{1}, \ldots, i_{p}\right\}$. By homology decomposition (4),

$$
H_{2}\left(\mathcal{R}_{K}\right) \cong \bigoplus_{J \subseteq[m]} \widetilde{H}_{1}\left(K_{J}\right)
$$

Since $K_{I}$ is a $p$-cycle, we have $\widetilde{H}_{1}\left(K_{I}\right)=\mathbb{Z}$. Because any subcomplex $K_{J}$ with $J \neq I$ is contractible, $\widetilde{H}_{1}\left(K_{J}\right)=0$ for $J \neq I$. It follows that $H_{2}\left(\mathcal{R}_{K}\right)=\mathbb{Z}$. 


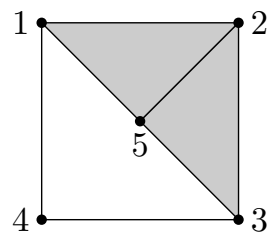

(a)

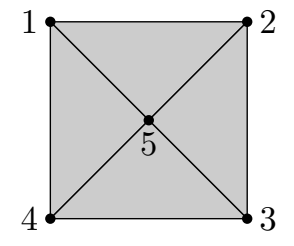

(b)

FIGURE 1.

(b) $\Rightarrow$ (c). Suppose $H_{2}\left(\mathcal{R}_{K}\right)=\mathbb{Z}$. Then only one summand in (11) is $\mathbb{Z}$, and all other summands are zero. Since $K$ is a flag complex, this implies that there exists a set of vertices $I=\left\{i_{1}, \ldots, i_{p}\right\}$ such that $K_{I}$ is a $p$-cycle with $p \geqslant 4$. Since $\widetilde{H}_{1}\left(K_{J}\right)=0$ for any proper subset $J \subseteq I$, any two vertices which are not adjacent in the $p$-cycle are not connected by an edge. If there exists a vertex $j \notin I$ in the complex $K$, then $\widetilde{H}_{1}\left(K_{I \cup\{j\}}\right)=0$ implies that the vertex $j$ is connected to each vertex in the $p$-cycle $I$. If $K$ has two vertices $j_{1}, j_{2} \notin I$ which are not connected by an edge, then the subcomplex $K_{\left\{i_{1}, i_{3}\right\} \cup\left\{j_{1}, j_{2}\right\}}$ is a 4 -cycle and $\widetilde{H}_{1}\left(K_{\left\{i_{1}, i_{3}\right\} \cup\left\{j_{1}, j_{2}\right\}}\right)=\mathbb{Z}$, which contradicts the assumption. Hence, all vertices of $K$ which are not in the set $I$ are connected to each other and to all vertices of $I$. Since $K$ is a flag complex, we obtain $K=C_{p} * \Delta^{q}$ for some $p \geqslant 4$ and $q \geqslant 0$.

(c) $\Rightarrow$ (a). First let $K$ be a $p$-cycle $C_{p}$. In this case the complex $\mathcal{R}_{K}$ is homeomorphic to a closed orientable surface of genus $(p-4) 2^{p-3}+1$ (see [5, Proposition 4.1.8]). Also, $\pi_{1}\left(\mathcal{R}_{K}\right) \cong R C_{K}^{\prime}$. Hence, $R C_{K}^{\prime}$ is a one-relator group.

Now let $\widetilde{K}=K * \Delta^{q}$, where $K$ is a $p$-cycle. Then $\mathcal{R}_{\widetilde{K}}=\mathcal{R}_{K} \times D^{q+1}$ and $R C_{\widetilde{K}}^{\prime}=\pi_{1}\left(\mathcal{R}_{\widetilde{K}}\right)=\pi_{1}\left(\mathcal{R}_{K}\right)=R C_{K}^{\prime}$ is a one-relator group.

(a) $\Rightarrow$ (b). Since $\mathcal{R}_{K}$ is an aspherical finite cell complex, the group $\pi_{1}\left(\mathcal{R}_{K}\right)$ is torsion-free (for example, see [9, Proposition 2.45]). So if $\pi_{1}\left(\mathcal{R}_{K}\right)=F / R$ is a one-relator group with a relation $r$, then $r$ is not a proper power $u^{n}$ for $n>1$, as otherwise the element $u$ would be of finite order.

Consider the space $Y\left(R C_{K}^{\prime}\right)$, constructed as in (10). According to the Lyndon Identity Theorem, $Y\left(R C_{K}^{\prime}\right)$ is homotopy equivalent to $K\left(R C_{K}^{\prime}, 1\right)$, so its homology groups coincide with the homology groups of the space $\mathcal{R}_{K}$. Proposition 3.1 implies that $H_{2}\left(\mathcal{R}_{K}\right)$ is either $\mathbb{Z}$ or 0 . The group $R C_{K}^{\prime}$ is not free, so the graph $K^{1}$ is not chordal, that is, there exists a chordless cycle on $I$ of length $p \geqslant 4$. Therefore, one of the summands on the right hand side of (11) is equal to $\mathbb{Z}=\widetilde{H}_{1}\left(K_{I}\right)$. Thus, $H_{2}\left(\mathcal{R}_{K}\right)=\mathbb{Z}$.

It remains to prove that (c) implies that $H_{k}\left(\mathcal{R}_{K}\right)=0$ for $k \geqslant 3$. Considering homology decomposition (4),

$$
H_{k}\left(\mathcal{R}_{K}\right) \cong \bigoplus_{J \subseteq[m]} \widetilde{H}_{k-1}\left(K_{J}\right)
$$

we claim that all summands with $k \geqslant 3$ on the right hand side are equal to 0 . Indeed, let $I=\left\{i_{1}, \ldots, i_{p}\right\}$ be the set of vertices of $K$ forming a $p$-cycle. Then $\widetilde{H}_{k-1}\left(K_{I}\right)=0$ for $k \geqslant 3$. Since any full subcomplex $K_{J}$ with $J \neq I$ is contractible, we get $\widetilde{H}_{k-1}\left(K_{J}\right)=0$. Hence, $H_{k}\left(\mathcal{R}_{K}\right)=0$ for $k \geqslant 3$.

The following examples illustrate Theorem 3.2.

\section{Example 3.3.}

1. Let $K$ be the flag complex in Figure 1 (a). Generator set (9) for the commutator 
subgroup $R C_{K}^{\prime}$ is

$$
\left(g_{3}, g_{1}\right),\left(g_{4}, g_{2}\right),\left(g_{5}, g_{4}\right),\left(g_{2},\left(g_{5}, g_{4}\right)\right) .
$$

These satisfy the relations

$$
\left(g_{3}, g_{1}\right)^{-1}\left(g_{4}, g_{2}\right)^{-1}\left(g_{3}, g_{1}\right)\left(g_{4}, g_{2}\right)=1, \quad\left(g_{3}, g_{1}\right)^{-1}\left(g_{5}, g_{4}\right)^{-1}\left(g_{3}, g_{1}\right)\left(g_{5}, g_{4}\right)=1
$$

and

$$
\left(g_{3}, g_{1}\right)^{-1}\left(g_{2},\left(g_{5}, g_{4}\right)\right)^{-1}\left(g_{3}, g_{1}\right)\left(g_{2},\left(g_{5}, g_{4}\right)\right)=1 \text {. }
$$

Indeed, since each of $g_{1}$ and $g_{3}$ commutes with each of $g_{2}$ and $g_{4}$, the commutators $\left(g_{4}, g_{2}\right)^{-1}$ and $\left(g_{3}, g_{1}\right)$ commute too. We therefore obtain

$$
\left(g_{3}, g_{1}\right)^{-1}\left(g_{4}, g_{2}\right)^{-1}\left(g_{3}, g_{1}\right)\left(g_{4}, g_{2}\right)=\left(g_{3}, g_{1}\right)^{-1}\left(g_{3}, g_{1}\right)\left(g_{4}, g_{2}\right)^{-1}\left(g_{4}, g_{2}\right)=1 \text {. }
$$

The other two relations are proved similarly. Using homology decomposition (4), we get $H_{2}\left(\mathcal{R}_{K}\right)=\mathbb{Z}^{3}$.

2. Let $K$ be the flag complex in Figure 1 (b). Generator set (9) for $R C_{K}^{\prime}$ is

$$
\left(g_{3}, g_{1}\right),\left(g_{4}, g_{2}\right)
$$

which satisfy a single relation $\left(g_{3}, g_{1}\right)^{-1}\left(g_{4}, g_{2}\right)^{-1}\left(g_{3}, g_{1}\right)\left(g_{4}, g_{2}\right)=1$. Here $R C_{K}^{\prime}$ is a one-relator group and $H_{2}\left(\mathcal{R}_{K}\right)=\mathbb{Z}$.

\section{Connected Sums of Sphere Products}

Let $M=\#_{i=1}^{k}\left(S^{d_{i}} \times S^{d-d_{i}}\right)$, where $d_{i} \geqslant 2, d \geqslant 4$ and \# denotes the connected sum operation on manifolds. Topologically, such connected sums are obtained by attaching a single cell to a wedge of spheres, that is, there is a cofibration sequence

$$
S^{d-1} \stackrel{w}{\longrightarrow} \bigvee_{i=1}^{k} S^{d_{i}} \vee S^{d-d_{i}} \stackrel{i}{\longrightarrow} \#_{i=1}^{k}\left(S^{d_{i}} \times S^{d-d_{i}}\right)
$$

where $w$ is the sum of Whitehead products $w_{i}: S^{d-1} \rightarrow S^{d_{i}} \vee S^{d-d_{i}}$. Denote by $\bar{M}$ the wedge $\bigvee_{i=1}^{d} S^{d_{i}} \vee S^{d-d_{i}}$. Then by the Bott-Samelson theorem $H_{*}(\Omega \bar{M}) \cong$ $T\left(a_{1}, b_{1}, \ldots, a_{k}, b_{k}\right)$, where $\operatorname{deg}\left(a_{i}\right)=d_{i}-1$ and $\operatorname{deg}\left(b_{i}\right)=d-d_{i}-1$. The looped inclusion $\Omega i: \Omega \bar{M} \rightarrow \Omega M$ induces a map of algebras

$$
(\Omega i)_{*}: T\left(a_{1}, b_{1}, \ldots, a_{k}, b_{k}\right) \longrightarrow H_{*}(\Omega M) .
$$

The adjoint $\bar{w}: S^{d-2} \rightarrow \Omega\left(\bigvee_{i=1}^{k} S^{d_{i}} \vee S^{d-d_{i}}\right)$ of the sum of Whitehead products $w$ induces a map $\bar{w}_{*}: H_{d-2}\left(S^{d-2}\right) \rightarrow H_{d-2}(\Omega \bar{M})$ which sends the canonical generator to the element $\chi=\left[a_{1}, b_{1}\right]+\cdots+\left[a_{k}, b_{k}\right]$. In particular, $\chi$ is primitive and $(\Omega i)_{*}(\chi)=$ 0 in $H_{*}(\Omega M)$. Then the algebra

$$
\frac{T\left(a_{1}, b_{1}, \ldots, a_{k}, b_{k}\right)}{\left\langle\left[a_{1}, b_{1}\right]+\cdots+\left[a_{k}, b_{k}\right]\right\rangle}
$$

is a primitively generated Hopf algebra, where the quotient ideal is two-sided, and the algebra map $(\Omega i)_{*}$ factors as a map of Hopf algebras

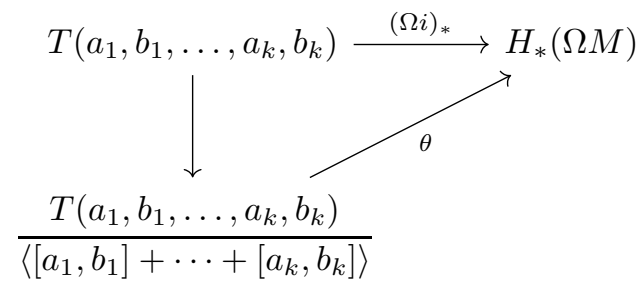

defining the map $\theta$.

The loop homology Hopf algebra $H_{*}(\Omega X ; \mathbb{Z})$ of a simply connected CW-complex $X$ can be calculated as homology of the cobar construction Cobar $C_{*}(X)$ of the 
reduced singular chains $C_{*}(X)[1]$, or as homology of the Adams-Hilton model [2] based on cells and attaching maps.

The cobar construction Cobar is a functor

$$
\text { Cobar: } \mathrm{DGC}_{1} \longrightarrow \text { DGA }
$$

from the category $\mathrm{DGC}_{1}$ of simply connected differential graded (dg) coalgebras to dg algebras. It assigns to a dg coalgebra $(C, \partial)$ with $C_{0}=\mathbb{Z}$ and $C_{1}=0$ the $\mathrm{dg}$ algebra

$$
\text { Cobar } C=(F(C), d)
$$

where $F(C)=T\left(s^{-1} \bar{C}\right)$ is the free associative algebra on the desuspended module $\bar{C}=C / \mathbb{Z}$, the cokernel of the coaugmentation $\mathbb{Z} \rightarrow C$. The differential $d$ is given by

$$
d c=-\partial c+\sum_{i=2}^{p-2}(-1)^{i} \Delta_{i, p-i} c
$$

where $c \in s^{-1} \bar{C}_{p}$ with comultiplication $\Delta c=c \otimes 1+1 \otimes c+\sum_{i=2}^{p-2} \Delta_{i, p-i} c$.

Adams [1] proved that for a simply connected CW-complex $X$ there is an isomorphism of Hopf algebras

$$
H_{*}(\Omega X) \cong H\left(\operatorname{Cobar} C_{*}(X), d\right)=\operatorname{Cotor}_{C_{*}(X)}(\mathbb{Z}, \mathbb{Z})
$$

where $C_{*}(X)$ is the reduced singular chain coalgebra of $X$.

The Adams-Hilton model [2] is a smaller dg algebra $A H_{*}(X)$ quasi-isomorphic to Cobar $C_{*}(X)$; it has generators corresponding to the cells of $X$ and differential defined via the attaching maps.

The following statement generalises [2, Corollary 2.4].

Proposition 4.1. For $d_{i} \geqslant 2$ and $d \geqslant 4$, there is an isomorphism of Hopf algebras

$$
H_{*}\left(\Omega\left(\#_{i=1}^{k} S^{d_{i}} \times S^{d-d_{i}}\right)\right) \cong \frac{T\left(a_{1}, b_{1}, \ldots, a_{k}, b_{k}\right)}{\left\langle\left[a_{1}, b_{1}\right]+\cdots+\left[a_{k}, b_{k}\right]\right\rangle}
$$

where $\operatorname{deg} a_{i}=d_{i}-1, \operatorname{deg} b_{i}=d-d_{i}-1$, and $\left[a_{i}, b_{i}\right]=a_{i} \otimes b_{i}+(-1)^{\operatorname{deg} a_{i} \operatorname{deg} b_{i}+1} b_{i} \otimes a_{i}$ is the graded commutator.

Proof. We consider the Adams-Hilton model of $M=\#_{i=1}^{k} S^{d_{i}} \times S^{d-d_{i}}$. The cofibration sequence (12) gives a CW-structure on $M$ consisting of cells $e^{0}, e_{i}^{d_{i}}, e_{i}^{d-d_{i}}$, $1 \leqslant i \leqslant k$, each attached trivially, and a single cell $e^{d}$ attached by the sum of Whitehead products $w_{i}: S^{d-1} \rightarrow S^{d_{i}} \vee S^{d-d_{i}}$. The Adams-Hilton model $A H_{*}(M)$ can be identified with the cobar construction on the coalgebra generated by positivedimensional cells, in which the differential is zero, $e_{i}^{d_{i}}$ and $e_{i}^{d-d_{i}}$ are primitives, and

$$
\Delta e^{d}=e^{d} \otimes 1+1 \otimes e^{d}+\sum_{i=1}^{k}\left(e_{i}^{d_{i}} \otimes e_{i}^{d-d_{i}}+(-1)^{d_{i}\left(d-d_{i}\right)} e_{i}^{d-d_{i}} \otimes e_{i}^{d_{i}}\right) .
$$

The Adams-Hilton model is therefore

$$
A H_{*}(M)=\left(T\left(a_{1}, b_{1}, \ldots, a_{k}, b_{k}, z\right), d\right)
$$

where $a_{i}=(-1)^{d_{i}} s^{-1} e_{i}^{d_{i}}, b_{i}=s^{-1} e_{i}^{d-d_{i}}, z=s^{-1} e^{d}$ and $\operatorname{deg} a_{i}=d_{i}-1, \operatorname{deg} b_{i}=$ $d-d_{i}-1$ and $\operatorname{deg} z=d-1$. Differential (15) is given by $d\left(a_{i}\right)=d\left(b_{i}\right)=0$ and

$$
\begin{aligned}
d(z) & =\sum_{i=1}^{k}\left((-1)^{d_{i}} s^{-1} e_{i}^{d_{i}} \otimes s^{-1} e_{i}^{d-d_{i}}+(-1)^{d-d_{i}}(-1)^{d_{i}\left(d-d_{i}\right)} s^{-1} e_{i}^{d-d_{i}} \otimes s^{-1} e_{i}^{d_{i}}\right) \\
& =\sum_{i=1}^{k}\left(a_{i} \otimes b_{i}+(-1)^{\left(d_{i}+1\right)\left(d-d_{i}\right)+d_{i}} b_{i} \otimes a_{i}\right)=\sum_{i=1}^{k}\left[a_{i}, b_{i}\right] .
\end{aligned}
$$


A nonzero $x \in A H_{*}(M)$ is a cycle if and only if $x$ is not in the two-sided ideal $\langle z\rangle$, and $x$ is a boundary if and only if $x \in\langle d(z)\rangle$. Therefore, homology of $\Omega M$ is as stated.

For a graded vector space $V$, denote by $P(V ; t)$ the Poincaré series of $V$.

Proposition 4.2. There is the following identity for the Poincaré series

$$
P\left(H_{*}\left(\Omega\left(\#_{i=1}^{k} S^{d_{i}} \times S^{d-d_{i}}\right) ; t\right)\right)=\frac{1}{1-\sum_{i=1}^{k}\left(t^{d_{i}-1}+t^{d-d_{i}-1}\right)+t^{d-2}} .
$$

Proof. Let $A=H_{*}\left(\Omega\left(\#_{i=1}^{k} S^{d_{i}} \times S^{d-d_{i}}\right)\right)$. By Proposition 4.1, $A$ is the quotient of the free associative algebra on the graded set $S=\left\{a_{1}, b_{1}, \ldots, a_{k}, b_{k}\right\}$, where $\operatorname{deg} a_{i}=d_{i}-1$ and $\operatorname{deg} b_{i}=d-d_{i}-1$, by the two-sided ideal generated by the element

$$
\chi=\sum_{i=1}^{k}\left[a_{i}, b_{i}\right]=a_{1} b_{1}+(-1)^{\operatorname{deg} a_{1} \operatorname{deg} b_{1}+1} b_{1} a_{1}+\sum_{i=2}^{k}\left[a_{i}, b_{i}\right] .
$$

Let $B$ be the graded free monoid on $S$. Then $B_{n}$, the $n$th graded component of $B$, is a generating set for $A_{n}$, the $n$th graded component of $A$. For any monomial $x \in A \backslash\{1\}$, write $x=s y$ for some unique $s \in S$ and $y \in B_{n-\operatorname{deg} s}$. If $x=a_{1} b_{1} y^{\prime}$ then using relation $\chi$ we rewrite

$$
x=\left((-1)^{\operatorname{deg} a_{1} \operatorname{deg} b_{1}} b_{1} a_{1}-\sum_{i=2}^{k}\left[a_{i}, b_{i}\right]\right) y^{\prime} .
$$

Let $B_{n}^{\prime}$ be the set of all elements in $B_{n}$ which do not start with $a_{1} b_{1}$. By induction, $B_{n}^{\prime}$ is a minimal generating set for $A_{n}$. Define $c_{n}=\left|B_{n}^{\prime}\right|=\operatorname{rank} A_{n}$ for $n \geqslant 1, c_{n}=0$ for $n<0$, and $c_{0}=1$. From the above description, $c_{n}$ satisfies the recurrence formula

$$
c_{n}=\sum_{i=1}^{k}\left(c_{n-d_{i}+1}+c_{n-d+d_{i}+1}\right)-c_{n-d+2}
$$

for $n \geqslant 1$. Multiplying by $t^{n}$ and summing over $n>0$ gives

$$
\begin{aligned}
P(A ; t)-1 & =\sum_{n=1}^{\infty} c_{n} t^{n} \\
& =\sum_{n=1}^{\infty}\left(\sum_{i=1}^{k}\left(c_{n-d_{i}+1}+c_{n-d+d_{i}+1}\right)-c_{n-d+2}\right) t^{n} \\
& =\sum_{i=1}^{k} \sum_{n=2-d_{i}}^{\infty} c_{n} t^{n+d_{i}-1}+\sum_{i=1}^{k} \sum_{n=2-d+d_{i}}^{\infty} c_{n} t^{n+d-d_{i}-1}-\sum_{n=3-d}^{\infty} c_{n} t^{n+d-2} \\
& =\left(\sum_{i=1}^{k}\left(t^{d_{i}-1}+t^{d-d_{i}-1}\right)-t^{d-2}\right) \sum_{n=0}^{\infty} c_{n} t^{n} \\
& =\left(\sum_{i=1}^{k}\left(t^{d_{i}-1}+t^{d-d_{i}-1}\right)-t^{d-2}\right) P(A ; t)
\end{aligned}
$$

which is rearranged to give the claimed identity. 


\section{One-Relator Algebras}

An algebra is a one-relator algebra if it is not free and can be written as the quotient of a free associative algebra by a two-sided ideal generated by a single element.

We recall the bigraded decomposition (3) of the integral homology of the momentangle complex $\mathcal{Z}_{K}$.

Theorem 5.1. Let $K$ be a flag simplicial complex on $[m]$. The following conditions are equivalent:

(a) $H_{*}\left(\Omega \mathcal{Z}_{K}\right)$ is a one-relator algebra;

(b) $H_{2-j, 2 j}\left(\mathcal{Z}_{K}\right)= \begin{cases}\mathbb{Z} & \text { if } j=p \text { for some } p, 4 \leqslant p \leqslant m \\ 0 & \text { otherwise; }\end{cases}$

(c) $K=C_{p}$ or $K=C_{p} * \Delta^{q}$ for $p \geqslant 4$ and $q \geqslant 0$, where $C_{p}$ is a p-cycle and $\Delta^{q}$ is a q-simplex.

If any one of these conditions is met, we have $H_{-i, 2 j}\left(\mathcal{Z}_{K}\right)=0$ for $j-i \geqslant 3$.

To prove the Theorem, we start by showing that if $K$ is a flag complex which is not of the form given in (c), then either $H_{*}\left(\Omega \mathcal{Z}_{K}\right)$ is free, or it has at least two relations. The following result gives a condition for $H_{*}\left(\Omega \mathcal{Z}_{K}\right)$ to have at least two relations.

Lemma 5.2. Let $K$ be a simplicial complex and suppose that $K_{I}$ and $K_{J}$ are distinct full subcomplexes of $K$ such that both $H_{*}\left(\Omega \mathcal{Z}_{K_{I}}\right)$ and $H_{*}\left(\Omega_{\mathcal{Z}_{K_{J}}}\right)$ have at least one relation. Then $H_{*}\left(\Omega \mathcal{Z}_{K}\right)$ is not a one-relator algebra.

Proof. Note that each of $\mathcal{Z}_{K_{I}}$ and $\mathcal{Z}_{K_{J}}$ retracts off $\mathcal{Z}_{K}$ as $K_{I}$ and $K_{J}$ are full subcomplexes. Therefore each of $\Omega \mathcal{Z}_{K_{I}}$ and $\Omega \mathcal{Z}_{K_{J}}$ retracts off $\Omega \mathcal{Z}_{K}$ and we obtain a commutative diagram of algebras

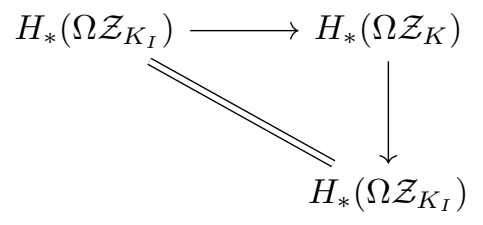

and similarly for $K_{J}$. In particular, each relation of $H_{*}\left(\Omega \mathcal{Z}_{K_{I}}\right)$ appears as a relation of $H_{*}\left(\Omega \mathcal{Z}_{K}\right)$ under the induced inclusion map and similarly for $K_{J}$, and the induced relations are distinct since $K_{I}$ and $K_{J}$ are.

Let $K$ be a simplicial complex on $[m]$. Suppose that $j \in K$ is a vertex and define the link

$$
\operatorname{lk}_{K}(j)=\{I \in K \mid j \cup I \in K, j \notin I\}
$$

and the star

$$
\operatorname{st}_{K}(j)=\{I \in K \mid j \cup I \in K\}=\operatorname{lk}_{K}(j) * j
$$

and assume that $\operatorname{lk}_{K}(j)$ is on the first $l$ vertices of $K$. Decompose $K=\operatorname{st}_{K}(j) \cup_{\mathrm{lk}_{K}(j)}$ $K_{[m] \backslash j}$. Then there is a homotopy pushout of moment-angle complexes

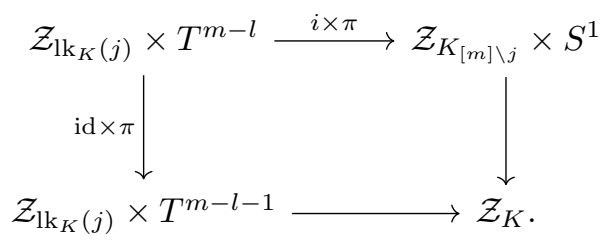


Lemma 5.3. If the map $i: \mathcal{Z}_{\operatorname{lk}_{K}(j)} \rightarrow \mathcal{Z}_{K_{[m] \backslash j}}$ is nullhomotopic, then there is a homotopy equivalence

$$
\mathcal{Z}_{K} \simeq \Sigma^{2}\left(\mathcal{Z}_{\operatorname{lk}_{K}(j)} \times T^{m-l-1}\right) \vee\left(\mathcal{Z}_{[m] \backslash j} \rtimes S^{1}\right) .
$$

Here the half-smash $X \rtimes Y$ of pointed spaces is defined by $X \times Y /(p t \times Y)$.

Proof. This is a particular case of [8, Lemma 3.3].

The following result shows that when $\mathcal{Z}_{[m] \backslash j}$ has the homotopy type of a connected sum of sphere products, $H_{*}\left(\Omega \mathcal{Z}_{K}\right)$ is not a one-relator algebra.

Lemma 5.4. Suppose that $M=\#_{i=1}^{k}\left(S^{d_{i}} \times S^{d-d_{i}}\right)$ where $d_{i} \geqslant 2$ and $d \geqslant 4$. Then $H_{*}\left(\Omega\left(M \rtimes S^{1}\right)\right)$ is not a one-relator algebra.

Proof. As in Proposition 4.1, we apply the Adams-Hilton model. A cell structure on $M \rtimes S^{1}$ is given by the image under the quotient map $M \times S^{1} \rightarrow M \rtimes S^{1}$, and therefore consists of cells $e^{0}, e_{i}^{d_{i}}, e_{i}^{d-d_{i}}, e_{i}^{d_{i}+1}, e_{i}^{d-d_{i}+1}, 1 \leqslant i \leqslant k$, along with two cells $e^{d}$ and $e^{d+1}$. The Adams-Hilton model $A H_{*}\left(M \rtimes S^{1}\right)$ can be identified with the cobar construction on the coalgebra generated by positive-dimensional cells, in which the differential is zero, $e_{i}^{d_{i}}, e_{i}^{d-d_{i}}, e_{i}^{d_{i}+1}, e_{i}^{d-d_{i}+1}$ are primitives, $\Delta e^{d}$ is given by (16) and

$$
\begin{array}{r}
\Delta e^{d+1}=e^{d+1} \otimes 1+1 \otimes e^{d+1}+\sum_{i=1}^{k}\left(e_{i}^{d_{i}} \otimes e_{i}^{d-d_{i}+1}+(-1)^{d_{i}\left(d-d_{i}+1\right)} e_{i}^{d-d_{i}+1} \otimes e_{i}^{d_{i}}\right. \\
\left.+(-1)^{d-d_{i}} e_{i}^{d_{i}+1} \otimes e_{i}^{d-d_{i}}+(-1)^{d_{i}\left(d-d_{i}\right)} e_{i}^{d-d_{i}} \otimes e_{i}^{d_{i}+1}\right) .
\end{array}
$$

The Adams-Hilton model is therefore given by

$$
A H_{*}\left(M \rtimes S^{1}\right)=\left(T\left(a_{1}, b_{1}, x_{1}, y_{1}, \ldots, a_{k}, b_{k}, x_{k}, y_{k}, z, w\right), d\right)
$$

where we set $a_{i}=(-1)^{d_{i}} s^{-1} e_{i}^{d_{i}}, b_{i}=s^{-1} e_{i}^{d-d_{i}}, x_{i}=(-1)^{d+1} s^{-1} e_{i}^{d_{i}+1}, y_{i}=$ $s^{-1} e_{i}^{d-d_{i}+1}, z=s^{-1} e^{d}, w=s^{-1} e^{d+1}$, so that $\operatorname{deg} a_{i}=d_{i}-1, \operatorname{deg} b_{i}=d-d_{i}-1$, $\operatorname{deg} x_{i}=d_{i}, \operatorname{deg} y_{i}=d-d_{i}, \operatorname{deg} z=d-1, \operatorname{deg} w=d$. Differential (15) is given by $d\left(a_{i}\right)=d\left(b_{i}\right)=d\left(x_{i}\right)=d\left(y_{i}\right)=0$,

$$
d(z)=\sum_{i=1}^{k}\left[a_{i}, b_{i}\right]
$$

and

$$
\begin{aligned}
d(w) & =\sum_{i=1}^{k}\left((-1)^{d_{i}} s^{-1} e_{i}^{d_{i}} \otimes s^{-1} e_{i}^{d-d_{i}+1}+(-1)^{d-d_{i}+1}(-1)^{d_{i}\left(d-d_{i}+1\right)} s^{-1} e_{i}^{d-d_{i}+1} \otimes s^{-1} e_{i}^{d_{i}}\right. \\
+ & \left.(-1)^{d_{i}+1}(-1)^{d-d_{i}} s^{-1} e_{i}^{d_{i}+1} \otimes s^{-1} e_{i}^{d-d_{i}}+(-1)^{d-d_{i}}(-1)^{d_{i}\left(d-d_{i}\right)} s^{-1} e_{i}^{d-d_{i}} \otimes s^{-1} e_{i}^{d_{i}+1}\right) \\
& =\sum_{i=1}^{k}\left(a_{i} \otimes y_{i}+(-1)^{\left(d_{i}+1\right)\left(d-d_{i}+1\right)+d_{i}} y_{i} \otimes a_{i}+x_{i} \otimes b_{i}+(-1)^{d_{i}\left(d-d_{i}+1\right)+1} b_{i} \otimes x_{i}\right) \\
& =\sum_{i=1}^{k}\left(\left[a_{i}, y_{i}\right]+\left[x_{i}, b_{i}\right]\right) .
\end{aligned}
$$

Therefore any element in $\langle d(z)\rangle$ or $\langle d(w)\rangle$ is trivial in homology since it is a boundary. This induces two relations in $H_{*}\left(\Omega\left(M \rtimes S^{1}\right)\right)$, as claimed.

Proof of Theorem 5.1. (c) $\Rightarrow$ (a). Suppose that $K=C_{p}$ or $K=C_{p} * \Delta^{q}$ for $p \geqslant 4$, $q \geqslant 0$. Since $\mathcal{Z}_{K}$ is homotopy equivalent to a connected sum of sphere products (5), the implication follows from Proposition 4.1.

(a) $\Rightarrow$ (c). Suppose that $K$ is a flag complex on $[m]$ such that $H_{*}\left(\Omega \mathcal{Z}_{K}\right)$ is a one-relator algebra. If $K^{1}$ is a chordal graph, then $\mathcal{Z}_{K}$ has the homotopy type of a 
wedge of spheres [7, Theorem 4.6], and thus $H_{*}\left(\Omega \mathcal{Z}_{K}\right)$ is a graded free associative algebra, which is a contradiction.

Therefore assume that $K^{1}$ is not chordal. In particular, there exists a set of vertices $I \subseteq[m]$ such that the full subcomplex $K_{I}$ is a $p$-cycle, and we enumerate $I=\left\{b_{1}, b_{2}, \ldots, b_{p}\right\}$. If $I=[m]$, that is $K=C_{p}$, then $H_{*}\left(\Omega \mathcal{Z}_{K}\right)$ is a one-relator algebra by Proposition 4.1.

Assume that $[m] \backslash I \neq \emptyset$. First, we show that each $j \in[m] \backslash I$ is connected to each vertex in $I$. Consider the full subcomplex $K_{I \cup j}$ of $K$, and observe that

$$
K_{I \cup j}=K_{I} \cup_{\mathrm{lk}_{I \cup j}(j)} \operatorname{st}_{I \cup j}(j) .
$$

Suppose that $K_{I \cup j} \neq K_{I} * j$. Since $K$ is flag, there exists $b_{l} \in I$ such that there is no edge from $j$ to $b_{l}$. Form the sequence of adjacent vertices $b_{l+1}, b_{l+2}, \ldots, b_{l+n_{1}}$, with the convention that $b_{p+1}=b_{1}$, where $n_{1} \geqslant 1$ is the smallest index such that there is an edge from $j$ to $b_{l+n_{1}}$. Similarly, form sequence of adjacent vertices $b_{l-1}, b_{l-2}, \ldots, b_{l-n_{2}}$, where $n_{2} \geqslant 1$ is again the smallest index such that there is an edge from $j$ to $b_{l-n_{2}}$. We consider four cases.

(i) Assume that there are no indices $n_{1}$ and $n_{2}$ as described above. In this case, there are no edges between $j$ and any vertex in $I$, and so $\operatorname{lk}_{I \cup j}(j)=\emptyset$. Then (17) takes the form

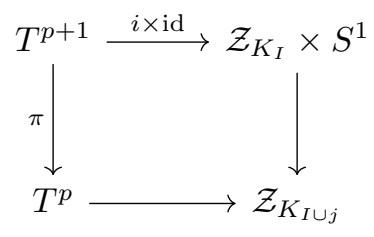

where the map $i: T^{p} \rightarrow \mathcal{Z}_{K_{I}}$ is nullhomotopic and therefore $\mathcal{Z}_{K_{I \cup j}} \simeq \Sigma^{2} T^{p} \vee\left(Z_{K_{I}} \rtimes\right.$ $\left.S^{1}\right)$ by Lemma 5.3. Since $\mathcal{Z}_{K_{I}}$ is homeomorphic to a connected sum of sphere products, Lemma 5.4 gives that $H_{*}\left(\Omega\left(\mathcal{Z}_{K_{I}} \rtimes S^{1}\right)\right)$ is not a one-relator algebra, and hence neither is $H_{*}\left(\Omega \mathcal{Z}_{K_{I \cup j}}\right)$.

(ii) If $b_{l+n_{1}}=b_{l-n_{2}}$, then $\operatorname{lk}_{I \cup j}(j)=b_{l+n_{1}}$, and $\mathcal{Z}_{K_{I \cup j}} \simeq \Sigma^{2} T^{p-1} \vee\left(Z_{K_{I}} \rtimes S^{1}\right)$ by Lemma 5.3. Thus $H_{*}\left(\Omega \mathcal{Z}_{K_{I \cup j}}\right)$ is not a one-relator algebra.

(iii) When $b_{l+n_{1}}$ and $b_{l-n_{2}}$ are adjacent in $K_{I}$, the link $\operatorname{lk}_{I \cup j}(j)=\left\{\left(b_{l+n_{1}}, b_{l-n_{2}}\right)\right\}$, and so $\mathcal{Z}_{K_{I \cup j}} \simeq \Sigma^{2} T^{p-2} \vee\left(Z_{K_{I}} \rtimes S^{1}\right)$ by Lemma 5.3. Thus $H_{*}\left(\Omega \mathcal{Z}_{K_{I \cup j}}\right)$ is not a one-relator algebra.

(iv) Finally, let $b_{l+n_{1}}$ and $b_{l-n_{2}}$ be distinct and not adjacent in $K_{I}$. Then by construction the full subcomplex $K_{\left\{j, b_{l-n_{2}}, \ldots, b_{l-1}, b_{l}, b_{l+1}, \ldots, b_{l+n_{1}}\right\}}$ of $K$ is a $\left(n_{1}+n_{2}+\right.$ 2)-cycle, which is distinct from $K_{I}$. Therefore by Lemma $5.2, H_{*}\left(\Omega \mathcal{Z}_{K_{I \cup j}}\right)$ is not a one-relator algebra.

In all of the above cases, since the full subcomplex $K_{I \cup j}$ retracts off $K$ and $H_{*}\left(\Omega \mathcal{Z}_{K_{I \cup j}}\right)$ is not a one-relator algebra, then neither is $H_{*}\left(\Omega \mathcal{Z}_{K}\right)$. This is a contradiction. We therefore conclude that $j$ is connected to each vertex in $K_{I}$ and therefore $K_{I \cup j}=K_{I} * j$.

Second, we show that if $j_{1}, j_{2} \in[m] \backslash I$, then $j_{1}$ and $j_{2}$ are connected by an edge. If not, since both $j_{1}$ and $j_{2}$ are connected to each vertex in $I$, then the full subcomplex $K_{\left\{j_{1}, b_{i_{1}}, j_{2}, b_{i_{3}}\right\}}$ is a 4-cycle distinct from the $p$-cycle $K_{I}$. Therefore, Lemma 5.2 implies that since $H_{*}\left(\Omega \mathcal{Z}_{I \cup\left\{j_{1}, j_{2}\right\}}\right)$ is not a one-relator algebra, neither is $H_{*}\left(\Omega \mathcal{Z}_{K}\right)$, which is a contradiction.

Therefore any vertex in $[m] \backslash I$ is connected to every vertex in $I$ and to every other vertex in $[m] \backslash I$. Since $K$ is flag, $K=K_{I} * \Delta^{q}$ for some $q \geqslant 0$. 
(c) $\Rightarrow$ (b). Suppose that $K=C_{p}$ or $K=C_{p} * \Delta^{q}$ for $p \geqslant 4$ and $q \geqslant 0$. Let the $p$-cycle $C_{p}$ of $K$ be supported on the set of vertices $I=\left\{b_{1}, \ldots, b_{p}\right\}$. By (3),

$$
H_{2-j, 2 j}\left(\mathcal{Z}_{K}\right) \cong \bigoplus_{J \subseteq[m],|J|=j} \widetilde{H}_{1}\left(K_{J}\right) .
$$

Since $K_{I}$ is a $p$-cycle, we have $\widetilde{H}_{1}\left(K_{I}\right)=\mathbb{Z}$. Because any subcomplex $K_{J}$ with $J \neq I$ is contractible, $\widetilde{H}_{1}\left(K_{J}\right)=0$ for $J \neq I$. It follows that $H_{2-p, 2 p}\left(\mathcal{Z}_{K}\right)=\mathbb{Z}$ and $H_{2-j, 2 j}\left(\mathcal{Z}_{K}\right)=0$ for $j \neq p$.

(b) $\Rightarrow(\mathrm{c})$. Suppose that $H_{2-j, 2 j}\left(\mathcal{Z}_{K}\right)$ is as described in (b). Then only one summand on the right hand side of (18) is $\mathbb{Z}$, and all other summands are zero. The same argument as in the proof of implication $(\mathrm{b}) \Rightarrow(\mathrm{c})$ of Theorem 3.2 shows that $K=C_{p} * \Delta^{q}$ for some $p \geqslant 4$ and $q \geqslant 0$.

It remains to prove that if $K=C_{p}$ or $K=C_{p} * \Delta^{q}$ for $q \geqslant 0$ then $H_{-i, 2 j}\left(\mathcal{Z}_{K}\right)=0$ for $j-i \geqslant 3$. Considering bigraded decomposition (3),

$$
H_{-i, 2 j}\left(\mathcal{Z}_{K}\right) \cong \bigoplus_{J \subseteq[m],|J|=j} \tilde{H}_{j-i-1}\left(K_{J}\right) .
$$

We claim that all summands on the right hand side with $j-i \geqslant 3$ are equal to 0 . Indeed, let $I=\left\{b_{1}, \ldots, b_{p}\right\}$ be the set of vertices of $K$ forming a $p$-cycle, $p \geqslant 4$. Then $\widetilde{H}_{j-i-1}\left(K_{I}\right)=0$ for $j-i \geqslant 3$. Since any full subcomplex $K_{J}$ with $J \neq I$ is contractible, we get $\widetilde{H}_{j-i-1}\left(K_{J}\right)=0$. Hence, $H_{-i, 2 j}\left(\mathcal{Z}_{K}\right)=0$ for $j-i \geqslant 3$.

The following examples illustrate Theorem 5.1.

\section{Example 5.5.}

1. Let $K$ be the flag complex in Figure 1 (a). Generator set (8) for $H_{*}\left(\Omega \mathcal{Z}_{K}\right)$ is

$$
\left[u_{3}, u_{1}\right],\left[u_{4}, u_{2}\right],\left[u_{5}, u_{4}\right],\left[u_{2},\left[u_{5}, u_{4}\right]\right] .
$$

These satisfy the relations

$$
\left[u_{3}, u_{1}\right]\left[u_{4}, u_{2}\right]-\left[u_{4}, u_{2}\right]\left[u_{3}, u_{1}\right]=0, \quad\left[u_{3}, u_{1}\right]\left[u_{5}, u_{4}\right]-\left[u_{5}, u_{4}\right]\left[u_{3}, u_{1}\right]=0
$$

and

$$
\left[u_{3}, u_{1}\right]\left[u_{2},\left[u_{5}, u_{4}\right]\right]-\left[u_{2},\left[u_{5}, u_{4}\right]\right]\left[u_{3}, u_{1}\right]=0
$$

which are derived by using the commutativity relations given in (7). By formula (3) we obtain $H_{-2,8}\left(\mathcal{Z}_{K}\right)=\mathbb{Z}^{2}$ and $H_{-3,10}\left(\mathcal{Z}_{K}\right)=\mathbb{Z}$. Hence, the homological condition of Theorem 5.1 (b) is not satisfied.

2. Let $K$ be the flag complex in Figure 1 (b). Generator set (8) for $H_{*}\left(\Omega \mathcal{Z}_{K}\right)$ is

$$
\left[u_{3}, u_{1}\right],\left[u_{4}, u_{2}\right]
$$

with a single relation $\left[u_{3}, u_{1}\right]\left[u_{4}, u_{2}\right]-\left[u_{3}, u_{1}\right]\left[u_{4}, u_{2}\right]=0$. Here $H_{*}\left(\Omega \mathcal{Z}_{K}\right)$ is a one-relator algebra, and formula $(3)$ gives $H_{-2,8}\left(\mathcal{Z}_{K}\right)=\mathbb{Z}$.

Recall that a simplicial complex $K$ is Golod if all cup products and higher Massey products vanish in $H^{*}\left(\mathcal{Z}_{K}\right)$. A simplicial complex $K$ is minimally non-Golod if $K$ is not Golod itself, but for every vertex $\{i\} \in K$ the deletion subcomplex $K-\{i\}=$ $K_{[m] \backslash\{i\}}$ is Golod. In the flag case, the properties of $H_{*}\left(\Omega \mathcal{Z}_{K}\right)$ being a one-relator algebra and $K$ being minimally non-Golod are related as follows.

Proposition 5.6. Let $K$ be a flag simplicial complex on $[m]$. The following conditions are equivalent:

(a) $\mathcal{Z}_{K}$ is homotopy equivalent to a connected sum of sphere products, with two spheres in each product;

(b) $H_{*}\left(\Omega \mathcal{Z}_{K}\right)$ is a one-relator algebra; 
(c) $K$ is minimally non-Golod, or $K=L * \Delta^{q}$ where $L$ is a minimally nonGolod complex and $q \geqslant 0$.

Proof. (a) $\Rightarrow$ (b). This follows from Proposition 4.1.

(b) $\Rightarrow$ (c). This follows from Theorem 5.1 , because a $p$-cycle $C_{p}$ with $p \geq 4$ is a minimally non-Golod complex.

(c) $\Rightarrow$ (a). This follows from the fact that if $K$ is minimally non-Golod and flag, then $K=C_{p}$ with $p \geqslant 4$. Indeed, let $K^{1}$ be one-skeleton of $K$. If $K^{1}$ is a chordal graph, then $\mathcal{Z}_{K}$ has the homotopy type of a wedge of spheres [7, Theorem 4.6], so $K$ is Golod and therefore not minimally non-Golod. Hence, $K$ contains a chordless cycle $C_{p}$ with $p \geqslant 4$. If $\{i\} \in K$ is a vertex not in $C_{p}$, then $K-\{i\}$ still contains a chordless cycle and therefore is not Golod. Therefore $K=C_{p}$, and the result follows from formula (5).

In the non-flag case the three properties in Proposition 5.6 are all different. The implication $(\mathrm{a}) \Rightarrow$ (c) holds in the non-flag case by a result of Amelotte [3, Theorem 1.2], and (a) $\Rightarrow(\mathrm{b})$ is Proposition 4.1. We illustrate the failure of other implications in the next example.

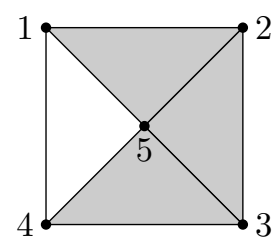

Figure 2.

Example 5.7. Let $K$ be the simplicial complex in Figure 2. A calculation similar to Proposition 4.1 using a cellular chain complex for $\mathcal{Z}_{K}$ shows that $H_{*}\left(\Omega \mathcal{Z}_{K}\right)$ is a one-relator algebra given by

$$
H_{*}\left(\Omega \mathcal{Z}_{K}\right) \cong \frac{T\left(a_{13}, a_{24}, b_{145}, b_{1452}, b_{1453}, b_{14523}\right)}{\left\langle\left[a_{13}, a_{24}\right]\right\rangle} .
$$

Moreover, a calculation similar to [5, Example 8.4.5] shows that the homomorphism $H_{*}\left(\Omega \mathcal{Z}_{K}\right) \longrightarrow H_{*}\left(\Omega D J_{K}\right)$ from (6) maps the generators $a_{13}, a_{24}, b_{145}, b_{1452}$, $b_{1453}, b_{14523}$ to the commutators $\left[u_{1}, u_{3}\right],\left[u_{2}, u_{4}\right],\left[u_{1}, u_{4}, u_{5}\right]$, [[u $\left.\left.u_{1}, u_{4}, u_{5}\right], u_{2}\right]$, $\left[\left[u_{1}, u_{4}, u_{5}\right], u_{3}\right],\left[\left[\left[u_{1}, u_{4}, u_{5}\right], u_{2}\right], u_{3}\right]$, respectively, where $\left[u_{1}, u_{4}, u_{5}\right]$ is the higher bracket corresponding to the missing face $\{145\}$.

Observe that $K$ is not minimally non-Golod, as $K-\{5\}$ is a 4-cycle, so implication (b) $\Rightarrow$ (c) of Proposition 5.6 fails in the non-flag case. Furthemore, (b) $\Rightarrow$ (a) also fails here, which is seen from the isomorphism of the cohomology ring of $\mathcal{Z}_{K}$ with that of $\left(S^{3} \times S^{3}\right) \vee S^{5} \vee S^{6} \vee S^{6} \vee S^{7}$.

The implication (c) $\Rightarrow$ (a) also fails in the non-flag case. Examples of minimally non-Golod complexes $K$ for which $\mathcal{Z}_{K}$ is not homotopy equivalent to a connected sum of sphere products were constructed by Limonchenko in [11, Theorem 2.6].

\section{REFERENCES}

[1] Adams, J. F. On the cobar construction. Proc. Nat. Acad. Sci. USA 42 (1956), 409-412.

[2] Adams, J. F.; Hilton, P. J. On the chain algebra of a loop space. Comment. Math. Helv. 30 (1956), 305-330.

[3] Amelotte, S. Connected sums of sphere products and minimally non-Golod complexes. Preprint (2020); arXiv:2006.16320. 
[4] Beben, P.; Wu, J. The homotopy type of a Poincaré duality complex after looping. Proc. Edinb. Math. Soc. (2) 58 (2015), no. 3, 581-616.

[5] Buchstaber, V.M.; Panov, T.E. Toric Topology. Mathematical Surveys and Monographs, vol. 204, American Mathematical Society, Providence, RI, 2015.

[6] Dyer, E.; Vasquez, A. T. Some small aspherical spaces. J. Austral. Math. Soc. 16 (1973), $332-352$

[7] Grbić J.; Panov T.; Theriault S.; Wu J. The homotopy types of moment-angle complexes for flag complexes. Trans. Amer. Math. Soc. 368 (2016), no. 9, 6663-6682.

[8] Grbić J.; Theriault S. The homotopy type of the complement of a coordinate subspace arrangement. Topology 46 (2007), no. 4, 357-396.

[9] Hatcher, A. Algebraic Topology. Cambridge University Press, Cambridge, 2002.

[10] Iriye, K.; Yano, T. A Golod complex with non-suspension moment-angle complex. Topology and its Applications 225 (2017), 145-163.

[11] Limonchenko I. Yu. Families of minimally non-Golod complexes and polyhedral products. Dal'nevost. Mat. Zh. 15 (2015), no. 2, 222-237 (in Russian); arXiv:1509.04302.

[12] Lyndon R.C. Cohomology theory of groups with a single defining relation. Ann. of Math. (2) 52 (1950), 650-665.

[13] McGavran, D. Adjacent connected sums and torus actions. Trans. Amer. Math. Soc. 251 (1979), 235-254.

[14] Panov T.E; Ray N. Categorical aspects of toric topology. Toric topology, Contemp. Math. 460, Amer. Math. Soc., Providence, RI, 2008, pp. 293-322.

[15] Panov T. E.; Veryovkin Ya. A. Polyhedral products and commutator subgroups of right-angled Artin and Coxeter groups. Sb. Math. 207 (2016), no. 11, 1582-1600.

[16] Veryovkin Ya. A. Pontryagin algebras of some moment-angle-complexes. Dal'nevost. Mat. Zh. (2016), no. 1, 9-23 (in Russian); arXiv:1512.00283.

School of Mathematical Sciences, University of Southampton, UK

E-mail address: j.grbic@soton.ac.uk

Department of Mathematics and Mechanics, Moscow State University, Russia

E-mail address: marina_ilyasova@bk.ru

Department of Mathematics and Mechanics, Moscow State University, Russia; Institute for Information Transmission Problems, Russian Academy of Sciences, MoSCOW, Russia;

Scientific Educational Mathematical Centre, Kazan Federal Univerisity, Russia

E-mail address: tpanov@mech.math.msu.su

School of Mathematical Sciences, University of Southampton, UK

E-mail address: g.j.h.simmons@soton.ac.uk 